\title{
Comparison of foot loading and foot strike pattern in women running in minimalist and conventional sports shoes
}

\author{
Soňa Jandová ${ }^{1,2, *}$, Jan Charousek ${ }^{3}$, and Miroslav Janura ${ }^{2}$ \\ ${ }^{I}$ Faculty of Mechanical Engineering, Technical University Liberec, Liberec, Czech Republic; ${ }^{2}$ Faculty of Physical Culture, \\ Palacký University Olomouc, Olomouc, Czech Republic; and ${ }^{3}$ Faculty of Science, Humanities and Education, Technical \\ University Liberec, Liberec, Czech Republic
}

Copyright: (C) 2019 S. Jandová et al. This is an open access article licensed under the Creative Commons Attribution License (https://creativecommons.org/licenses/by/4.0/).

Background: At present, most runners use conventional sports shoes to protect and absorb the impacts that may cause overloading of the feet and joints. On the other hand, some runners tend to run almost barefoot or in minimalist footwear that may lead to foot strike pattern changes. Objective: The aim of this study was to analyse and to compare the foot strike pattern and foot loading when running in conventional running shoes (CRS) and minimalist running shoes (MRS) in a group of recreational women runners. Methods: A group of 14 experienced recreational women runners participated in this research. A Pedar-X system (Novel, Munich, Germany) was used to analyse the interaction of the foot with the ground while running in different type of shoes from as it relates to vertical force and plantar pressure distribution. The participants ran over a distance of $100 \mathrm{~m}$ with their habitual foot strike pattern in minimalist and also in conventional running shoes and data was always obtained while running along a straight $50 \mathrm{~m}$ long section $\left(\mathrm{v}=3.33 \pm 0.2 \mathrm{~m} \cdot \mathrm{s}^{-1}\right)$. For assessment of the temporal and kinetics variables differences, the Wilcoxon test and the unpaired $t$-tests were used. Results: Recreational women runners showed statistically significant shift of the plantar pressures towards the forefoot when running in MRS compared to CRS $(p=.047)$. Duration of swing phase in MRS and in CRS was similar $(p=.291)$. When running in MRS, foot loading during initial contact was significantly higher than when running in CRS. Loading in the area of midfoot and forefoot was significantly higher in MRS too. Conclusions: Runners shifted their foot strike pattern forward when running in MRS; running in MRS may increase the foot loading and we recommend the use of minimalist footwear only after adequate foot strengthening and adaptation to avoid possible risk of foot injuries.

Keywords: pressure insole, kinetic, foot strike pattern, initial contact

\section{Introduction}

The most natural movement locomotion of humans are walking and running that are very popular in both recreational and competitive forms. People run on different types of surfaces, which require a specific shoe design. At present, most runners use conventional sports shoes to protect and absorb the impacts that may cause overloading of the foot and joints. On the other hand, the book "Born to run" (McDougall, 2009) started the recent popular trend of running barefoot or in minimalist footwear. Due to the increasing popularity of running in minimalist shoes, researchers

\footnotetext{
* Address for correspondence: Soňa Jandová, Department of Applied Mechanics, Faculty of Mechanical Engineering, Technical University of Liberec, Studentská 2, 46117 Liberec, Czech Republic.E-mail: sona.jandova@tul.cz
}

focussed on monitoring the footfall pattern when running in different footwear or barefoot (Ahn, Brayton, Bhatia, \& Martin, 2014; Hasegawa, Yamauchi, \& Kraemer, 2007; Hein \& Grau, 2014; Kasmer, Ketchum, \& Liu, 2014; Miller, Whitcome, Liebermann, Norton, \& Dyer, 2014; Larson et al., 2011).

Lieberman (2014) used a high speed video camera to determine the foot strike pattern. He measured the angle of incidence by measuring the orientation of the calcaneus and the 5th metatarsal head markers relative to the horizontal at the first frame of contact minus the same angle measured while sitting with boards placed under the heel and the phalanges and metatarsal heads. The foot strike patterns can also be identified by visual inspection of the 2D video sequence (Lieberman, 2014) and plantar pressure distribution in general can be evaluated using measuring insoles (Putti, Arnold, Cochrane, \& Abboud, 2007). 
According to previous studies (Hasegawa et al., 2007; Larson et al., 2011) $75-90 \%$ shod runners use rearfoot strike (RFS). Minimalist shoes have gained popularity recently because it is speculated to strengthen the foot muscles and foot arches, which may help prevent injuries (Chen, Sze, Davis, \& Cheung, 2016). According to previous studies (e.g., Jandová et al., 2018; Kasmer et al., 2014) minimalist shoes influence the footfall pattern when runners in minimalist shoes tend to shift footfall in the anterior-posterior direction towards forefoot. It is considered that runners over long distances run rather over the forefoot to eliminate large painful impacts in the heel area. These impacts can be absorbed while using sports shoes. Footfall pattern also influences ground reaction forces (Nigg, 2010). According to Whittle (1999), RFS causes significant impact and vertical ground reaction force increase in the first 20-50 ms of the stance phase. Willy and Davis (2014) reported greater foot loading while running in minimalist shoes than in standard shoes.

In previous studies (e.g., Deaner, 2013) sex differences in running physiology were described. In addition, Vannatta and Kernozek (2018) reported sex-related biomechanical factors in running, when men and women demonstrate differences in gluteal muscle forces and hip kinetics and kinematics during running. Since most of the previous studies dealt with foot strike pattern focused on the male category of runners (e.g., De Wit, De Clercq, \& Aerts, 2000; Willy \& Davis, 2014) or reported data without sex differences (Ahn et al., 2014; Hein \& Grau, 2014; Kasmer et al., 2014; Larson, 2014), this study focuses on a group of recreational women runners and the aim of this study was to analyse the footfall pattern, to measure the foot load and to compare it when running in conventional running shoes (CRS) and minimalist running shoes (MRS). We assume that in the given conditions, the footfall pattern while running in MRS and CRS will vary and we expect higher ground reaction forces acting while running in MRS.

\section{Methods}

\section{Participants}

A group of 14 experienced recreational women runners (mean age: $31.92 \pm 5.34$ years, mean body height: $1.69 \pm 0.07 \mathrm{~m}$, mean body mass: $63.31 \pm 4.89 \mathrm{~kg}$ ) engaged in running for more than one year participated in this research. The participants trained regularly, ran at least $30 \mathrm{~km}$ a week $(37.54 \pm 9.43 \mathrm{~km})$ and alternated between using MRS and CRS. The foot size requirement was EU 39-40 to be possible to use the same footwear for all participants (EU 39-40). The study was approved by the Institutional Review Board for testing in human subjects and was performed in accordance with the ethical standards of the Helsinki Declaration.

\section{Data collection}

The Pedar-X system (Novel, Munich, Germany) was used to analyse the interaction of the foot with the ground while running in different type of shoes as it relates to vertical force and plantar pressure distribution. The reaction force perpendicular to the surface was detected by measuring insoles with 99 sensors. This measuring system allows data to be recorded in $100 \mathrm{~Hz}$ frequency. Participants ran on artificial grass in both MRS (Vivobarefoot Primus) and CRS (Salomon XA PRO). All participants ran in the same pairs of shoes and for all measurements we used the same pair of measuring insole.

\section{Protocol}

The participants were first familiarized with the experiment protocol and subsequently signed an informed consent form. At the beginning of data collection, anthropometric data (body height, mass), and also age) was recorded. Data was collected after dynamic warming up (duration 10 minutes) and after speed standardization. The participants were asked to run $100 \mathrm{~m}$ at the required speed of $3.33 \mathrm{~m} \cdot \mathrm{s}^{-1}$ in their habitual footfall pattern. Data for the run was always obtained when running along a straight $50 \mathrm{~m}$ long section, $30 \mathrm{~m}$ from the start. The run in this $50 \mathrm{~m}$ section was measured and the average speed was calculated $\left(\mathrm{v}=3.33 \pm 0.2 \mathrm{~m} \cdot \mathrm{s}^{-1}\right)$. This was the same for both CRS and MRS. All participants ran the selected section 3 times in one type of shoes and 3 times in other type of shoes (randomly first in CRS or in MRS) with a pause of 5 minutes between each run to avoid fatigue. At the beginning of each run, the system for measuring the in-shoe pressure was calibrated when standing on one leg separately for each shoe in accordance with the manufacturer's recommendations.

\section{Data analysis}

The Pedar-X Recorder (Version 20.3.36; Novel, Munich, Germany) was used to analyse the following kinetic and temporal variables: maximal vertical force during initial phase (IC) acting between the foot and ground normalized to body weight $\left(\mathrm{relF}_{\max } \mathrm{IC}=\mathrm{F}_{\max } \mathrm{IC} / \mathrm{m} \cdot \mathrm{g}\right)$, maximal vertical force normalized to body weight during stance under the whole foot ( $\mathrm{relF}_{\max }$ ), under the heel ( $\left.\mathrm{relF}_{\max } \mathrm{HEEL}\right)$, under the midfoot (relF ${ }_{\text {max }}$ MIDFOOT) and forefoot $\left(\right.$ relF $_{\max }$ FOREFOOT) and duration of stance phase (s) and swing phase (s). The insoles were divided into 3 parts according to software masks - forefoot, midfoot, 
rearfoot. Foot strike pattern was determined on the basis of the COP position at initial contact (Breine, Malcolm, Frederick, \& De Clercq, 2014; Jandová et al., 2018). When the peak pressure during IC was under the forefoot, this footfall pattern was indicated as FFS. When the peak pressure during IC was under the midfoot, this footfall pattern was indicated as MFS and when the peak pressure during IC was under the rearfoot, this footfall pattern was indicated as RFS.

\section{Statistical analysis}

The Shapiro-Wilk test was used to verify normality. Given that some variables did not conform to normal distribution, a non-parametric Wilcoxon test was used for repeated measurements when testing the study hypothesis (evaluation of stance phase duration, swing phase duration, relF ${ }_{\max }, \mathrm{relF}_{\max } \mathrm{IC}$ ). Due to the fact that some runners had changed the footfall pattern when running in MRS and CRS, there was no paired data for the evaluation of each footfall type. For this reason, the unpaired $t$-test was used for evaluation of relF ${ }_{\max }$ HEEL, relF $_{\text {max }}$ MIDFOOT and relF ${ }_{\text {max }}$ FOREFOOT. Computed statistical values were considered significantly different when $p \leq .05$. Calculations were carried out using the software R (Version 3.4.2; R Foundation for Statistical Computing, Vienna, Austria).

\section{Results}

\section{Foot strike patterns}

On the basis of the measured pressure distribution values during IC in the individual parts, it was assessed whether the runners applied the FFS, MFS, or RFS pattern. According to the analysis of the dynamometric recording for the runners clad in CRS, 5 runners (36\%) used the MFS pattern and 9 runners (64\%) had the RFS pattern. When running in the minimalist shoes the foot strike pattern changed in 7 runners (50\%) and other runners used the same footfall pattern in both types of shoe. If there was a change, the foot strike pattern shifted from MFS to FFS or from RFS to MFS and in 1 runner it shifted from RFS to FFS. In summation, 5 out of 14 runners (36\%) in minimalist footwear used FFS, further 3 (21\%) relied on MFS, and the remaining 6 (43\%) utilized the RFS. As a result of this shift, a total of 4 runners using FFS when running in minimalist shoes demonstrated no contact of the heel with the ground throughout the gait cycle. This phenomenon appeared in selected subjects exclusively when wearing minimalist footwear. When using CRS, brief contact between the heel and ground was always observed even in runners using FFS (0.02-0.04 s). Thus, it is possible to say that in selected recreational women runners there was a statistically significant shift of the peak pressure towards the forefoot during IC when running in MRS compared to CRS ( $p=.047)$ and the trajectory of Centre of Pressure was closer to the forefoot.

\section{Stance and swing phase duration}

For the studied group of women runners, one-side alternative hypothesis that stance phase in MRS is shorter was not confirmed $(p=.998)$. The average values for both shoe types were very similar (CRS: $0.194 \pm 0.012 \mathrm{~s}$, MRS: $0.193 \pm 0.014 \mathrm{~s}$ ). The duration of swing phase in MRS and in CRS was very similar (CRS: $0.469 \pm 0.026 \mathrm{~s}$, MRS: $0.471 \pm 0.021 \mathrm{~s}$ ) and the differences were not significant $(p=.291)$.

When comparing the contact time between runners with different footfall pattern the contact time was the longest in case of RFS. When shifting, the landing towards the forefoot the contact time was shortened. Mean values for MRS were: RFS - $0.198 \pm 0.014 \mathrm{~s}$, MFS $-0.195 \pm 0.004 \mathrm{~s}$, and FFS $-0.188 \pm 0.008 \mathrm{~s}$. Stance duration in CRS was: RFS - $0.196 \pm 0.015 \mathrm{~s}$, MFS $-0.188 \pm 0.01 \mathrm{~s}$, and not applicable for FFS.

\section{Foot loading during stance phase}

Running in MRS and CRS was characterised by differences in foot loading during first contact with the ground and during the stance phase (Table 1). Wearing MRS during the initial phase (IC) resulted in the maximal vertical force values being significantly $(p=.002)$ higher when compared with CRS. When comparing the maximal vertical force normalized to body weight during stance phase under the whole foot $\left(\mathrm{relF}_{\max }\right)$ and under the heel ( $\mathrm{relF}_{\max }$ HEEL), no significant differences between MRS and CRS were found $(p>.05)$. In addition, significant differences were observed in normalized vertical force during stance phase under the midfoot (relF max $_{\text {MIDFOOT) and forefoot }}$ (relF ${ }_{\max }$ FOREFOOT) when the values in MRS were higher $(p<.001)$.

\section{Discussion}

The purpose of this study was to determine how minimalist shoes influence the footfall pattern and foot load compared to sports shoes in a group of women runners.

The main finding of the study was that the foot strike behaviour among recreational women runners was influenced by the type of shoes used and the different types of foot strike patterns lead to differences in foot loading in midfoot and forefoot. 
Table 1

Foot loading (normalized to body weight) while running in minimalist and conventional running shoes

\begin{tabular}{|c|c|c|c|c|c|}
\hline & \multicolumn{2}{|c|}{ MRS } & \multicolumn{2}{|c|}{ CRS } & \multirow[b]{2}{*}{$p$} \\
\hline & Mean & $S D$ & Mean & $S D$ & \\
\hline $\mathrm{relF}_{\max } \mathrm{IC}$ & 1.47 & 0.15 & 1.12 & 0.18 & .002 \\
\hline relF $_{\max }$ & 2.69 & 0.31 & 2.68 & 0.28 & .283 \\
\hline $\operatorname{relF}_{\max } \mathrm{HEEL}$ & 0.82 & 0.52 & 0.83 & 0.42 & .083 \\
\hline $\mathrm{relF}_{\max } \mathrm{MIDFOOT}$ & 1.37 & 0.25 & 1.33 & 0.20 & $<.001$ \\
\hline relF $_{\max }$ FOREFOOT & 1.50 & 0.31 & 1.39 & 0.22 & $<.001$ \\
\hline
\end{tabular}

Note. $\quad \mathrm{MRS}=$ minimalist running shoes; $\mathrm{CRS}=$ conventional running shoes; $\mathrm{relF}_{\max } \mathrm{IC}=$ maximal vertical force during initial phase; relF $_{\max }=$ maximal vertical force; $\mathrm{relF}_{\max }$ HEEL, MIDFOOT, FOREFOOT = maximal vertical force acting under the heel, midfoot or forefoot.

\section{Foot strike patterns}

In previous studies, foot strike pattern was assessed either on the basis of dynamographic record analysis (Breine et al., 2014; De Cock, Vanrenterghem, Willems, Witvrouw, \& De Clercq, 2008) or pursuant to analysis of video sequences, when authors measured the angle of incidence by measuring the orientation of the calcaneus and the 5th metatarsal head markers relative to the horizontal at the first frame of contact minus the same angle measured while sitting with boards placed under the heel and the phalanges and metatarsal heads (Lieberman, 2014). In our study three types of footfall patterns (forefoot strike, midfoot strike and rearfoot strike) were assessed according to pedography record while running in CRS and MRS. In our study comprising a group of 14 recreational women runners, 5 runners $(36 \%)$ used MFS when the ground is first touched by both the heel and the ball of the big toe simultaneously and 9 runners (64\%) had the RFS pattern while running in conventional running shoes. During RFS, runners hit the ground with the calcaneus first. In minimalist shoes, 5 runners (36\%) used FFS where the metatarsal heads on the lateral side touch the ground first. This ratio is slightly higher in percentage representation than in the study of Larson (2014) who stated that among minimally shod runners $33 \%$ were FFS runners. In our study, further 3 (21.4\%) runners used MFS and in the remaining 6 runners RFS was determined. In the case of RFS, after first contact with the ground under the heel, the pressure moved further to the middle part of the foot and then progressed to the metatarsal balls. A significantly lower number of runners used RFS when shod in CRS compared to other investigations (Hasegawa et al., 2007; Larson et al., 2011). This can be explained by the fact that the study was realised on artificial grass and this surface is softer than, for example, asphalt that is typical for road runs. That could have influenced their foot strike patterns (Nigg, 2010). In case of MFS, runners landed on the middle part of the foot first, most frequently on the outer part. Afterwards, the contact remained in the middle part, spread in mediolateral direction (inwardly), and then shifted towards the forefoot. Runners who used FFS and landed on the forefoot first shifted the plantar pressure towards the middle foot (mainly its outer part) and then in the direction of the forefoot again.

When running in minimalist shoes, the foot strike pattern shifted in anterior-posterior direction in 7 runners $(50 \%)$. These runners who ran with RFS in conventional running shoes changed the footfall to MFS and runners who used MFS in conventional running shoes used FFS in minimalist shoes. Only 1 runner changed the footfall pattern between RFS in conventional running shoes to FFS in minimalist shoes. An interesting trend was also monitored in the form of not using heel contact with the ground while running in MRS. When using CRS, brief contact of the heel with the ground was always observed even in runners using FFS (0.02-0.04 s).

In analysing the first foot contact, it is necessary to take into account the running technique and speed on a long track, which is different compared to runs over shorter distances (Larson, 2014; Lieberman, 2014; Kasmer et al., 2014; Miller et al., 2014; Pontzer et al., 2014). Hayes and Caplan (2012) reported different foot strike pattern for different running distance (800 m running distance reported ration for men: RFS $15 \%$, MFS $50 \%$, FFS $35 \%$ and for women: RFS $32 \%$. MFS $41 \%$, FFS $27 \%$; $1500 \mathrm{~m}$ running distance for men: RFS $26 \%$, MFS $37 \%$, FFS $37 \%$ and for women: RFS $33 \%$, MFS $42 \%$, FFS $25 \%$ ). With regards to these results and differences in gluteal muscle forces and hip kinetics and kinematics during running reported by Vannatta and Kernozek (2018), it is desirable to analyse running technique with respect to sex difference, but there is a lack of studies focusing on foot strike pattern only in women. 


\section{Stance and swing phase duration}

Based on previous studies (De Wit et al., 2000; Divert, Mornieux, Baur, Mayer, \& Belli, 2005), we expected shortening of the contact time with the ground in MRS. In our study, shortening of the stance phase in MRS or in CRS was not confirmed when the mean values for both shoe type were very similar. Running speed was almost the same while running in MRS and in CRS. Our result values are lower than the results of De Wit et al. (2000), Divert et al. (2005) or Francis, Ledingham, Clarke, Collins, and Jakeman (2016) who reported in a group of male runners longer stance duration in CRS than in MRS. These differences in our results can be explained by the differences in women and men running techniques when men run with longer strides (Cheuvront, Carter, De Ruisseau, \& Moffatt, 2005) and they apply greater ground forces than women (Weyand, Sternlight, Bellizzi, \& Wright, 2000).

When comparing the stance phase duration between runners with different footfall pattern it is clear that the stance phase is the longest in RFS (Deflandre, Schwartz, Weerts, Crosier, \& Bury, 2016) when the runners land on the heel and then the contact continues forward through midfoot to the forefoot. Mean values for MRS are: RFS $0.198 \pm 0.014 \mathrm{~s}$, MFS $0.195 \pm 0.004 \mathrm{~s}$, and FFS $0.188 \pm 0.008 \mathrm{~s}$. Stance duration in CRS is: RFS $0.196 \pm 0.015 \mathrm{~s}$, MFS $0.188 \pm 0.01 \mathrm{~s}$, and there was no case with FFS. Fellin, Rose, Royer, and Davis (2010) reported longer STANCE duration (0.26 s) while using RFS in sports shoes in similar running speed $\left(\mathrm{v}=3.35 \mathrm{~m} \cdot \mathrm{s}^{-1}\right)$.

\section{Foot loading during stance phase}

The changes in foot strike pattern caused differences in foot loading during first contact with the ground and during the stance phase. Rearfoot strike causes significant impact during the first 20-50 ms of stance phase and it also causes a very significant increase in ground reaction forces (Whittle, 1999). In our study, minimalist shoes resulted, during initial contact, in maximal vertical force values being significantly $(p=.002)$ higher when compared with CRS. This finding is in accordance with the study by Nigg (2010). In our study, the maximal vertical force normalized to body weight during initial contact was: $\mathrm{MRS}-\mathrm{relF}_{\max } \mathrm{IC}=1.47 \pm 0.15$, CRS $-\mathrm{relF}_{\max } \mathrm{IC}=1.12 \pm 0.18$. At the phase of initial contact of stance, FFS significantly increases activation of musculus gastrocnemius in contrast to RFS. For RFS, there is clear activation before contact with the ground because of stereotype gait comprising lifting of the toe and striking on the heel. Switching from RFS to FFS is possible by plantarflexion of the ankles and activating the calf muscles earlier and for longer than with RFS (Ahn et al., 2014).
When comparing maximal vertical force normalized to body weight during the stance phase under the whole foot $\left(\mathrm{relF}_{\max }\right)$ no significant differences between MRS and CRS were found. This finding is in accordance with previous studies (De Wit et al., 2000; Paquette, Zhang, \& Baumgartner, 2013); however, the study by De Wit et al. was realised with slightly higher speed $\left(\mathrm{v}=3.5 \mathrm{~m} \cdot \mathrm{s}^{-1}\right)$. On the other hand, Divert et al. (2005) reported significant differences between MRS and CRS, but in their study the running speed $\left(\mathrm{v}=3 \mathrm{~m} \cdot \mathrm{s}^{-1}\right)$ was slightly lower than in our study $\left(\mathrm{v}=3.33 \mathrm{~m} \cdot \mathrm{s}^{-1}\right)$.

When comparing maximal vertical force normalized to body weight during stance phase under the heel no significant differences between MRS and CRS were found. In addition, significant differences were observed in normalized vertical force during stance phase under the midfoot (relF max $_{\text {MIDFOOT) and }}$ forefoot ( $\mathrm{relF}_{\max }$ FOREFOOT) while using MRS. The construction of sports shoes helps with shock absorption in the heel whereas minimalist shoes absorb these impacts to a lesser degree. For those runners, who want to use MRS without any previous experience, the increased load on the foot muscles and calf musculature, especially if it occurs suddenly, could put the runner at risk for muscle soreness or foot and ankle injuries resulting from overuse. This further supports the need for proper training, strengthening and conditioning for a proper transition to an FFS pattern (Samaan, Rainbow, \& Davis, 2014).

Limitations of this study include the fact that the measurement was realised on a selected track that was shorter than what the recreational women runners normally used on regular basis. The reason for this was to ensure homogeneous conditions for all participants, which was very important for interpretation of the results. Due to the limited size of the study group, which consisted of 14 recreational women runners, any generalisation of the findings to all runners must be made carefully and we recommend using these results only when comparing women.

\section{Conclusion}

In conclusion, therefore, it can be emphasised that a change in the foot strike pattern occurs when running in minimalist shoes and conventional running shoes. Runners shifted their foot strike pattern forward when running in minimalist shoes. This trend was confirmed in $50 \%$ of monitored recreational women runners. These runners who ran with RFS in conventional running shoes changed the footfall to MFS and runners who used MFS in conventional running shoes used 
FFS in minimalist shoes. Runners in MRS using FFS tend to avoid heel contact with the ground. When running in MRS, foot loading during initial contact was significantly higher than when running in CRS. Loading in the area of midfoot and forefoot was significantly higher in MRS. The increased load on the foot muscles, especially if it occurs suddenly, could put the runner at risk for muscle soreness or foot and ankle injuries resulting from overuse and we recommend to use minimalist footwear only after adequate foot strengthening and adaptation.

\section{Conflict of interest}

There were no conflicts of interest.

\section{References}

Ahn, A. N., Brayton, C., Bhatia, T., \& Martin, P. (2014). Muscle activity and kinematics of forefoot and rearfoot strike runners. Journal of Sport and Health Science, 3, 102-112.

Breine, B., Malcolm, P., Frederick, E. C., \& De Clercq, D. (2014). Relationship between running speed and initial foot contact patterns. Medicine \& Science in Sports \& Exercise, 46, 1595-1603.

Chen, T. L.-W., Sze, L. K. Y., Davis, I. S., \& Cheung, R. T. H. (2016). Effects of training in minimalist shoes on the intrinsic and extrinsic foot muscle volume. Clinical Biomechanics, 36, 8-13.

Cheuvront, S. N., Carter, R., De Ruisseau, K. C., \& Moffatt, R. J. (2005). Running performance differences between men and women. Sports Medicine, 35, 1017-1024.

De Cock, A., Vanrenterghem, J., Willems, T., Witvrouw, E., \& De Clercq, D. (2008). The trajectory of the centre of pressure during barefoot running as a potential measure for foot function. Gait and Posture, 27, 669-675.

De Wit, B., De Clercq, D., \& Aerts, P. (2000). Biomechanical analysis of the stance phase during barefoot and shod running. Journal of Biomechanics, 33, 269-278.

Deaner, R. O. (2013). Physiology does not explain all sex differences in running performance. Medicine \& Science in Sports \& Exercise, 45, 146-147.

Deflandre, D., Schwartz, C., Weerts, J.-P., Croisier, J.-L., \& Bury, T. (2016). A comparison of 3D methods for identifying the stance phase in treadmill running for both rearfoot and forefoot runners. Journal of Sports Science, 4, 124-131.

Divert, C., Mornieux, G., Baur, H., Mayer, F., \& Belli, A. (2005). Mechanical comparison of barefoot and shod running. International Journal of Sports Medicine, 26, 593-598.

Fellin, R., Rose, W. C., Royer, T. D., \& Davis, I. S. (2010). Comparison of methods for kinematic identification of footstrike and toe-off during overground and treadmill running. Journal of Science and Medicine in Sport, 13, 646-650.

Francis, P., Ledingham, J., Clarke, S., Collins, D. J., \& Jakeman, P. (2016). A comparison of stride length and lower extremity kinematics during barefoot and shod running in well trained distance runners. Journal of Sports Science and Medicine, 15, 417-423.

Hasegawa, H., Yamauchi, T., \& Kraemer, W. J. (2007). Foot strike patterns of runners at the $15-\mathrm{km}$ point during an elite-level half marathon. Journal of Strength and Conditioning Research, 21, 888-893.

Hayes, P., \& Caplan, N. (2012). Foot strike patterns and ground contact times during high-calibre middle-distance races. Journal of Sports Sciences, 30, 1275-1283.

Hein, T., \& Grau, S. (2014). Can minimal running shoes imitate barefoot heel-toe running patterns? A comparison of lower leg kinematics. Journal of Sport and Health Science, 3, 67-73.

Jandová, S., Sparks, M., Olešniewicz, P., Charousek, J., Chrástková, M., \& Markiewicz, J. (2018). Changes in foot strike pattern and pressure distribution when running in minimalist and traditional running shoes. Medicina dello Sport, 71, 257-267.

Kasmer, M. E., Ketchum, N. C., \& Liu, X. C. (2014). The effect of shoe type on gait in forefoot strike runners during a 50-km run. Journal of Sport and Health Science, 3, 122-130.

Larson, P. (2014). Comparison of foot strike patterns of barefoot and minimally shod runners in a recreational road race. Journal of Sport and Health Science, 3, 137-142.

Larson, P., Higgins, E., Kaminski, J., Decker, T., Preble, J., Lyons, D., \& Normile, A. (2011). Foot strike patterns of recreational and sub-elite runners in a long-distance road race. Journal of Sports Sciences, 29, 1665-1673.

Lieberman, D. E. (2014). Strike type variation among Tarahumara Indians in minimal sandals versus conventional running shoes. Journal of Sport and Health Science, 3, 86-94.

McDougall, C. (2009). Born to run. New York, NY: Knopf Doubleday.

Miller, E. E., Whitcome, K. K., Lieberman, D. E., Norton, H. L., \& Dyer, R. E. (2014). The effect of minimal shoes on arch structure and intrinsic foot muscle strength. Journal of Sport and Health Science, 3, 74-85.

Nigg, B. M. (2010). Biomechanics of sports shoes. Calgary, Canada: Topline Printing.

Paquette, M. R., Zhang, S., \& Baumgartner, L. D. (2013). Acute effects of barefoot, minimal shoes and running shoes on lower limb mechanics in rear and forefoot strike runners. Footwear Science, 5, 9-18.

Pontzer, H., Suchman, K., Raichlen, D. A., Wood, B. M., Mabulla, A. Z. P., \& Marlowe, F. W. (2014). Foot strike patterns and hind limb joint angles during running in Hadza hunter-gatherers. Journal of Sport and Health Science, 3, 95-101.

Putti, A. B., Arnold, G. P., Cochrane, L., \& Abboud, R. J. (2007). The Pedar in-shoe system: Repeatability and normal pressure values. Gait and Posture, 25, 401-405.

Samaan, C. D., Rainbow, M. J., \& Davis, I. S. (2014). Reduction in ground reaction force variables with instructed barefoot running. Journal of Sport and Health Science, 3, 143-151.

Vannatta, C. N., \& Kernozek, T. W. (2018). Sex differences in gluteal muscle forces during running. Sports Biomechanics. 
Advance online publication. doi:10.1080/14763141.2018 .1548641

Weyand, P. G., Sternlight, D. B., Bellizzi, M. J., \& Wright, S. (2000). Faster top running speeds are achieved with greater ground forces not more rapid leg movements. Journal of Applied Physiology, 89, 1991-1999.
Whittle, M. W. (1999). Generation and attenuation of transient impulsive forces beneath the foot: A review. Gait and Posture, 10, 264-275.

Willy, R. W., \& Davis, I. S. (2014). Kinematic and kinetic comparison of running in standard and minimalist shoes. Medicine \& Science in Sports \& Exercise, 46, 318-323. 